\title{
Recent updates to the Arnold Mirror Modeler and integration into the evolving NASA overall design system for large space-based optical systems \\ William R. Arnold, Sr.*a \\ ${ }^{a}$ NASA Marshall Space Flight Center, Huntsville, Al, USA 35806
}

\begin{abstract}
Since last year, a number of expanded capabilities have been added to the modeler. The support the integration with thermal modeling, the program can now produce simplified thermal models with the same geometric parameters as the more detailed dynamic and even more refined stress models. The local mesh refinement and mesh improvement tools have been expanded and more user friendly.

The goal is to provide a means of evaluating both monolithic and segmented mirrors to the same level of fidelity and loading conditions at reasonable man-power efforts. The paper will demonstrate most of these new capabilities.
\end{abstract}

Keywords: Mirror Modeling, FEA, Large Space-based, Optical Systems

\section{INTRODUCTION}

For a number of years the Advanced Mirror Technology Development (AMTD) project has been developing design tools and validating manufacturing methods to support the coming generations of large spaced based telescopes.

The challenges of larger size mirrors and limited payload and shroud capacities (for both existing, under development or planned) have created the need for optimization of all aspects of the optics and instruments.

The Arnold Mirror Modeler is one of those tools intended to make the optimization problem more efficient by radically decreasing the time required to generate complex Finite Element models of very large egg-crate style lightweight mirrors and their suspension systems (both monolith and segmented)

\section{What does the modeler do?}

The modeler creates input decks for commercial Finite Element Analysis programs such as ANSYS, ABAQUS and NASTRAN. The modeler creates a complete analysis stream, including the model, loads [static and dynamic], plots and a summary file of input variable and results suitable for optimization or trade studies. The values of all settings in the program can be archived and recalled to continue or redo any configuration. Figure 1 shows the interface and a typical model, in this case loaded into ABAQUS. Various graphics windows (mesh pattern and 3D model) allow the user to refine and adjust the parameters before committing to an analysis run.

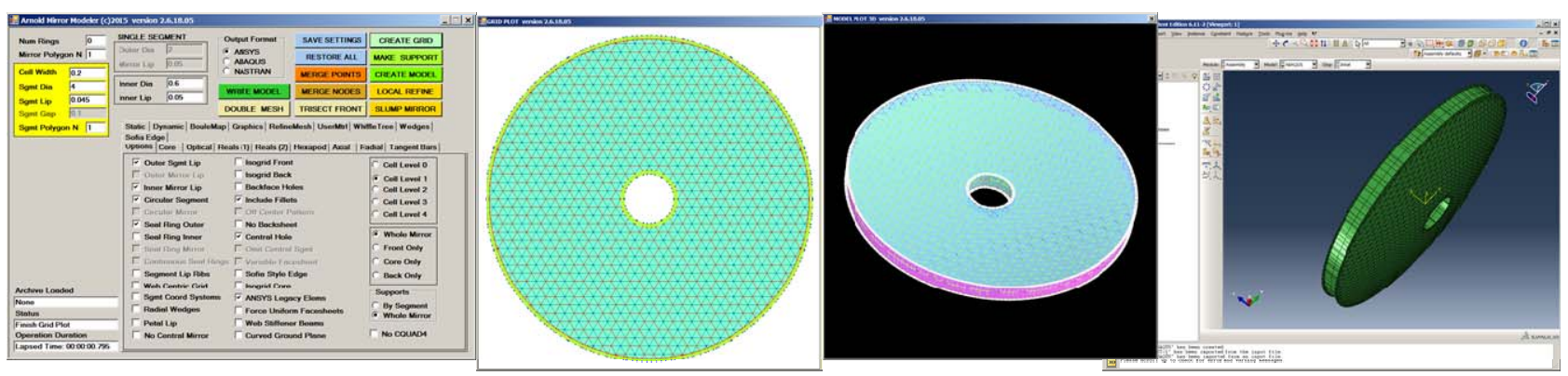

Figure 1 Typical Arnold Mirror Modeler Interface 


\section{Previous Capabilities}

The modeler is intended to be capable of modeling any known mirror material and construction method currently available or planned. Figure 2 shows some of the mirror configurations and suspension systems which can be created. For a more comprehensive listing of features of the earlier versions, see the references.
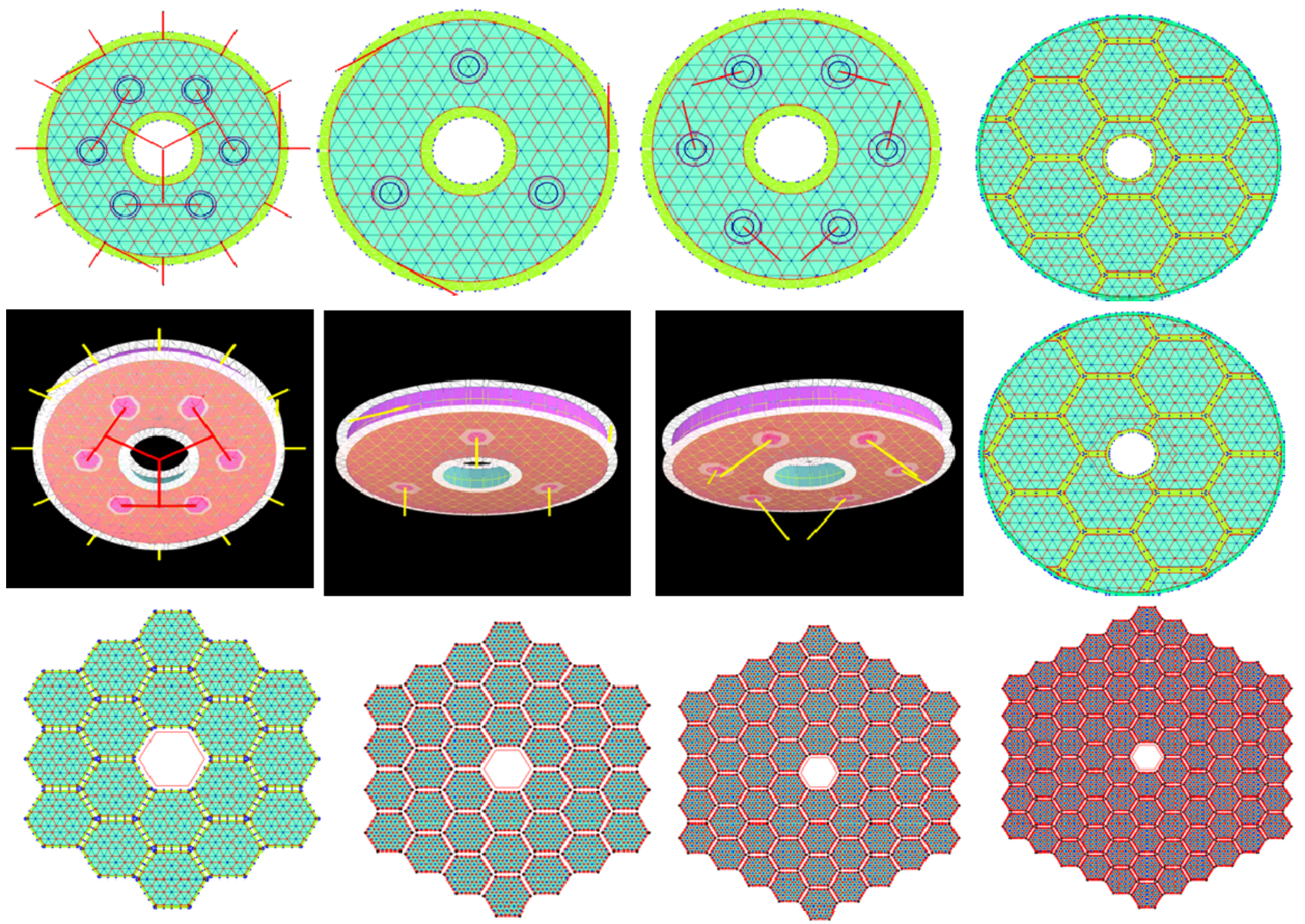

Figure 2 Representative mirror configuration possible with AMM 


\section{WHAT'S NEW IN THE CURRENT VERSION}

\section{SOFIA style mirror}

Since light-weighting Zerodur mirrors require milling, undercut pocket milling of the cells is the most risky operation. The largest outer lib which can be open back milled is desirable. The program allows varying number the major radial ribs as well as the number of minor ribs between each major rib. The depth of the median and edge beams can be varied as well. Both flat back (the original design) and meniscus or uniform depth mirrors are possible. The program calculates the volume of material that has to be removed.
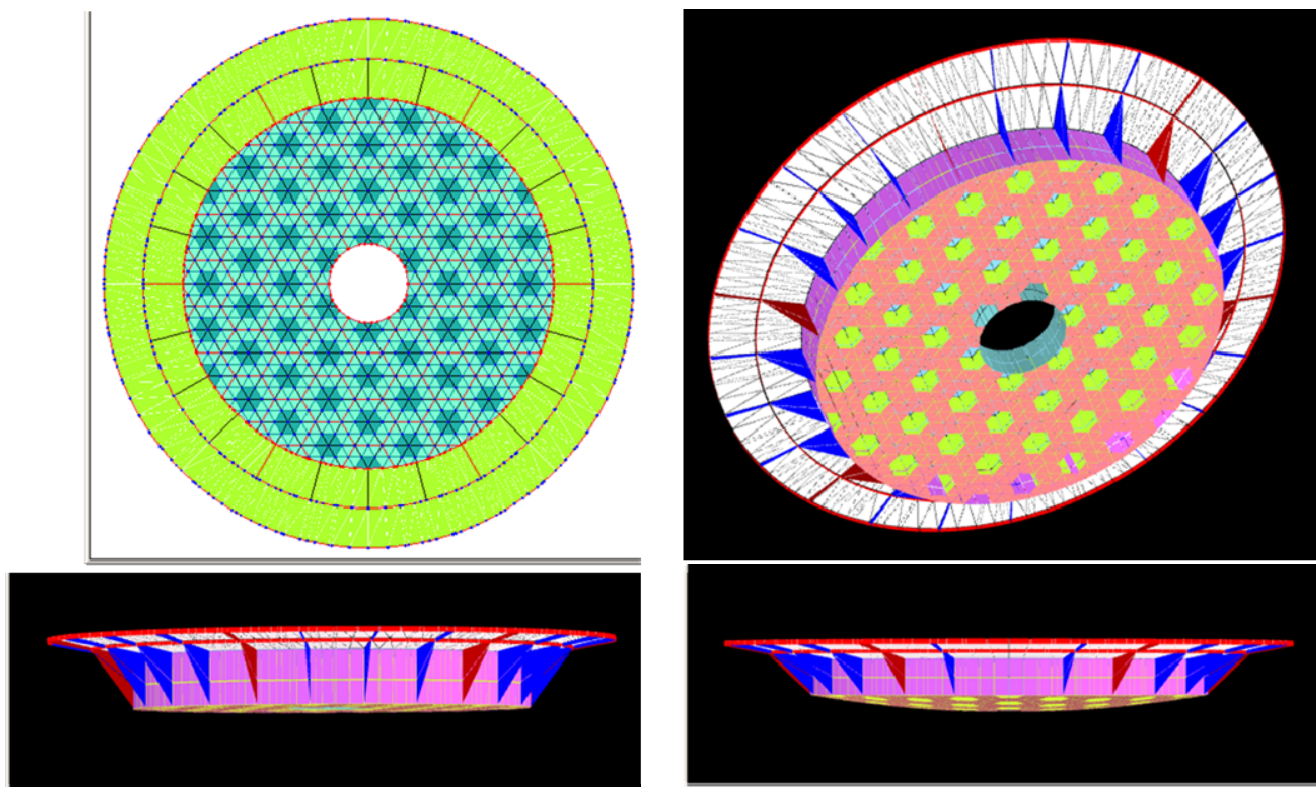

Figure 3 Both flat back and meniscus versions of Sofia style mirrors.

\section{Back edge (web) stiffener beams}

A stiffness improvement over straight open back pocketing of Zerodur is to initially make wide webs, then undercut mill a portion of the web thickness. This is less aggressive than SOFIA's pocketing, but can be beneficial. This detail is also suitable to silicon carbide and even beryllium designs.
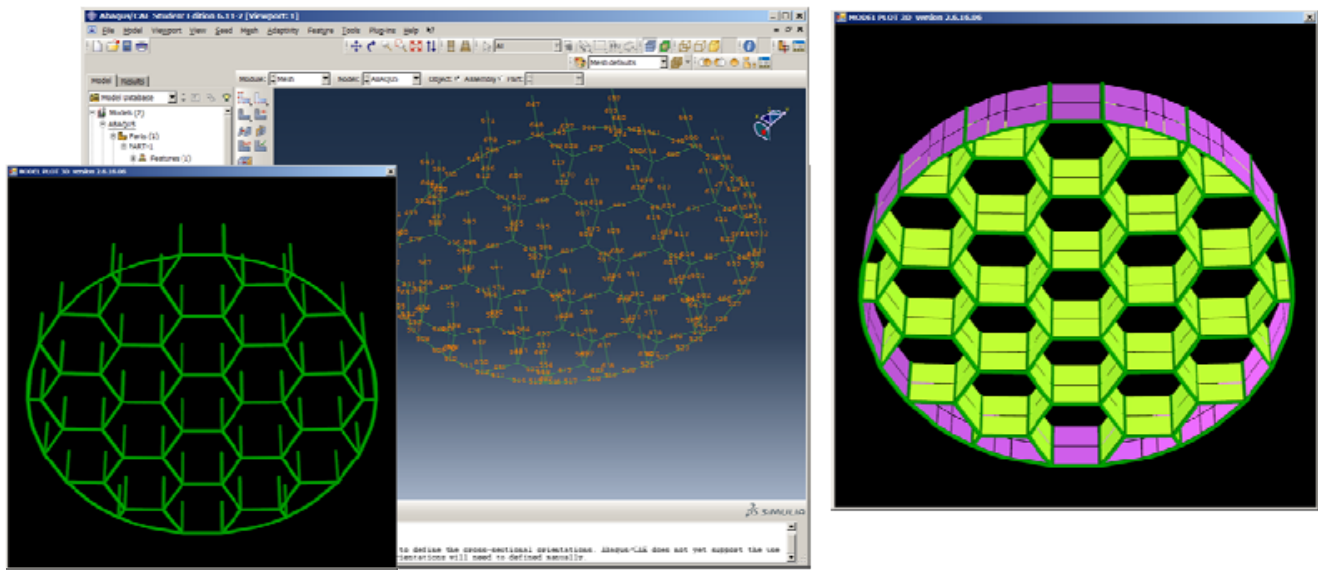

Figure 4 Edge beams for open back models 


\section{Wedge segments (also allows multiple rings)}

These can be merged into monolithic mirrors to simulate for example the diffusion bonding of silicon carbide mirrors or low temperature fusion bonding of ULE segments. The inner radius of the wedge can be circular as shown.in figure 5 or straight as shown in figure 6.
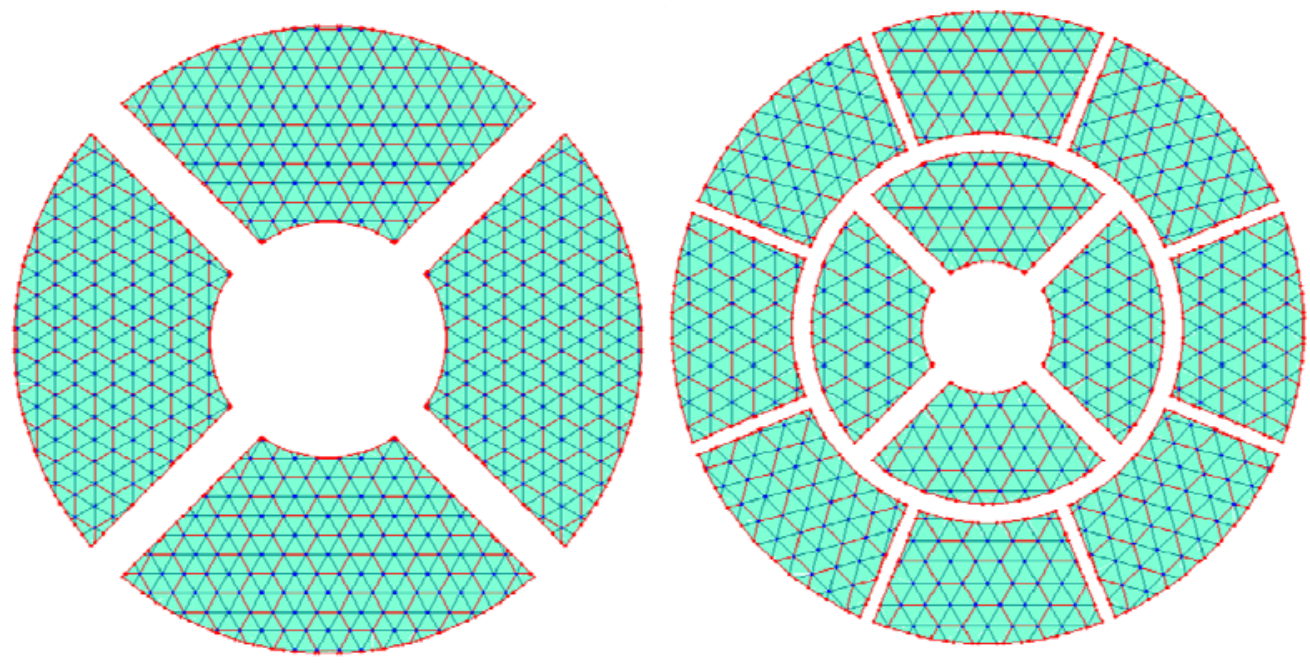

Figure 5 Wedges can be segments or merged into monolith mirror.

\section{Combining petals with monolith mirror}

One option for dealing with the problem of limited shroud diameter is to have as large a central monolith as possible, with deployable petals. This provides a better diffraction pattern than uniform segment patterns, as well as more mission flexibility.
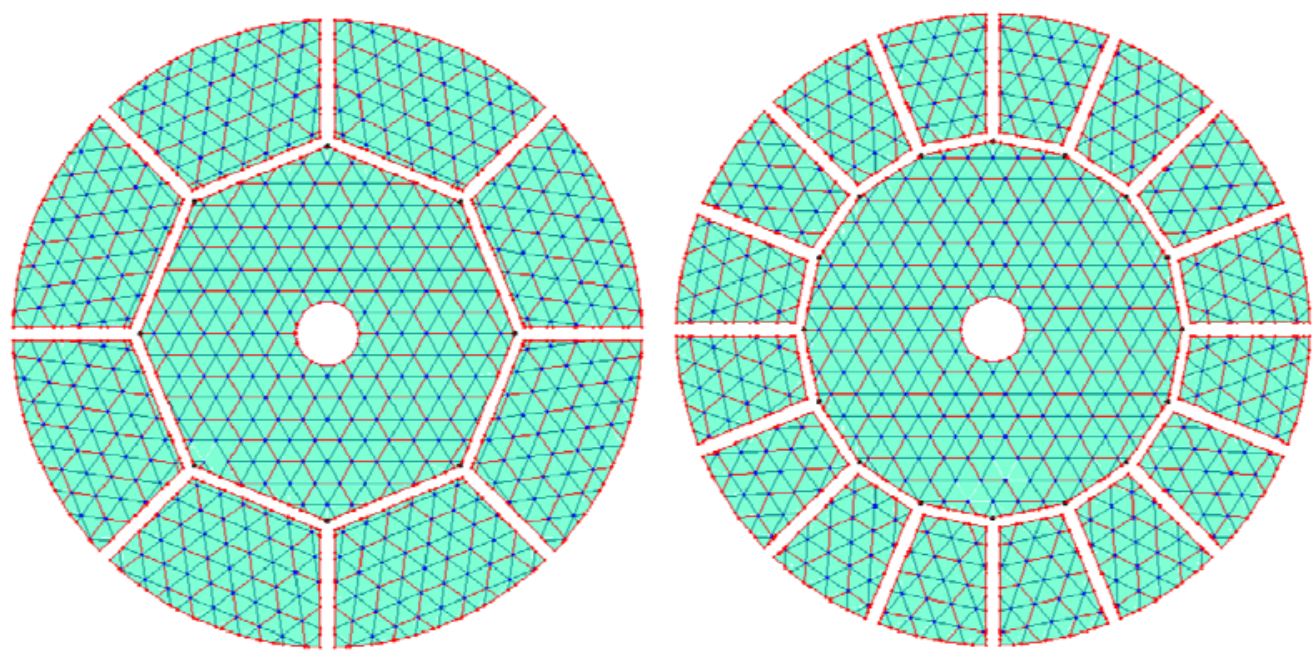

Figure 6 Combination of central monolith with radial petals. 


\section{N-polygon perimeters}

As a consequence of the petal or wedge capabilities, a generalized polygon perimeter shape is now supported. Up to $\mathrm{n}=16$ is possible. Both segment and whole mirror (merged segmented) perimeters can be configured. While not very practical for large mirrors, the model can also do flats and concave optics for special applications.
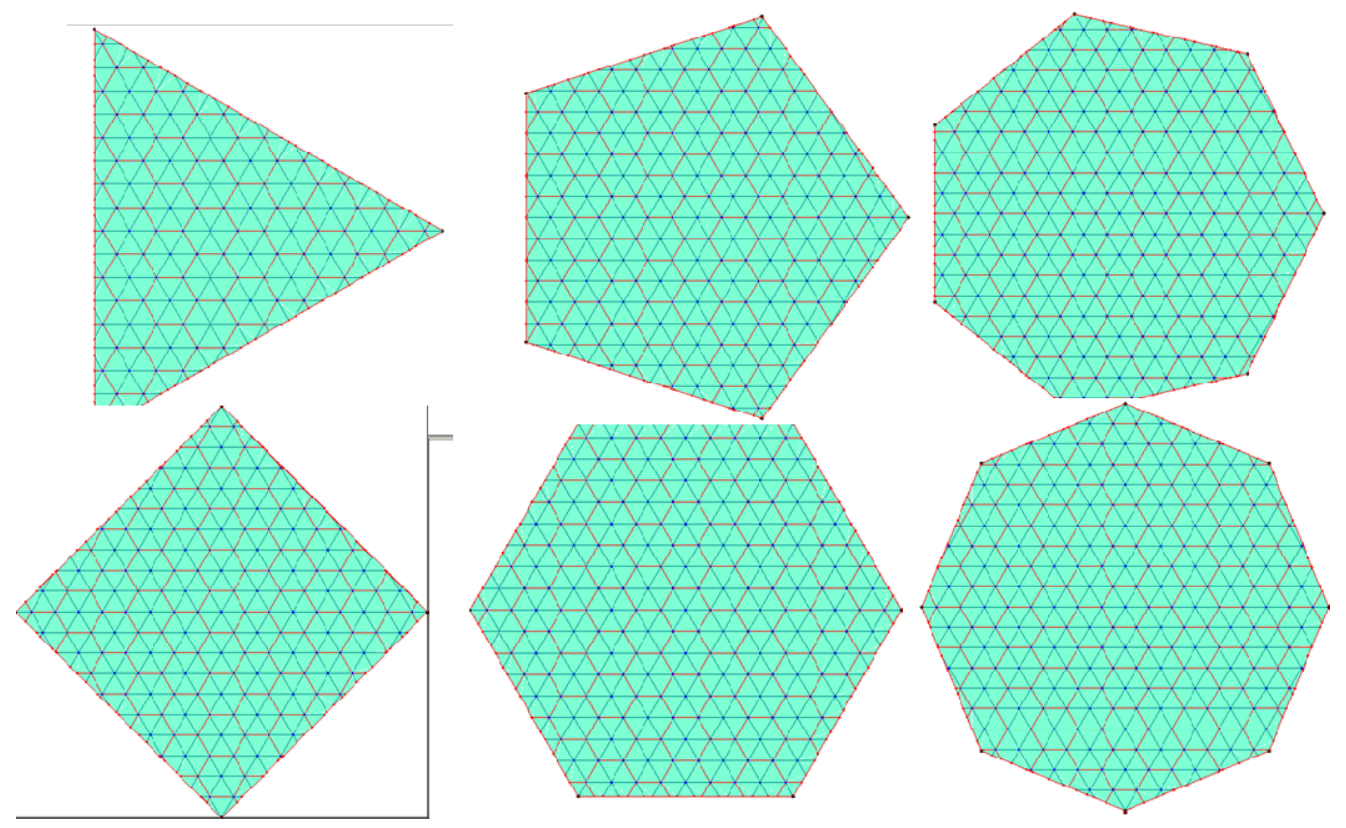

Figure 7 N-polygon shapes.

\section{Curved backplane definition}

As the segmented mirror sizes continue to grow, it becomes desirable to define the attachment plane for the segment support systems as a curve. This provides a more uniform strut stiffness for dynamic behavior of system These attachment points (number and coordinates are stored in a file for integration of the mirror model into a higher assembly model.
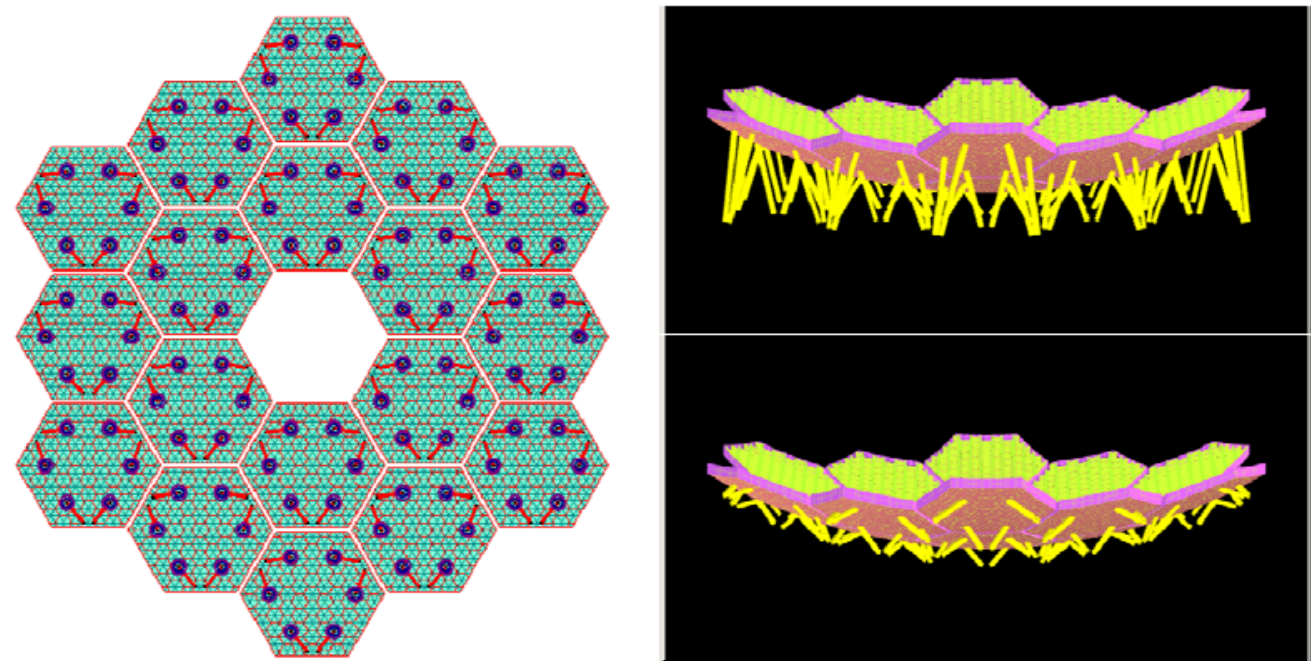

Figure 8 Backplane options, flat or curved. 


\section{Automatics repositioning of bond-pads}

One of the more .tedious tasks in matching up a suspension system support locations on the mirror to the stiffest spot originally required repeated adjustment of parameters and replotting the support system until they appear to be close. This was both inefficient and imprecise, so the option was added to let the program find the nearest cell center or web intersection and move the center of the pad to that position. The user has the choice of center or intersection.

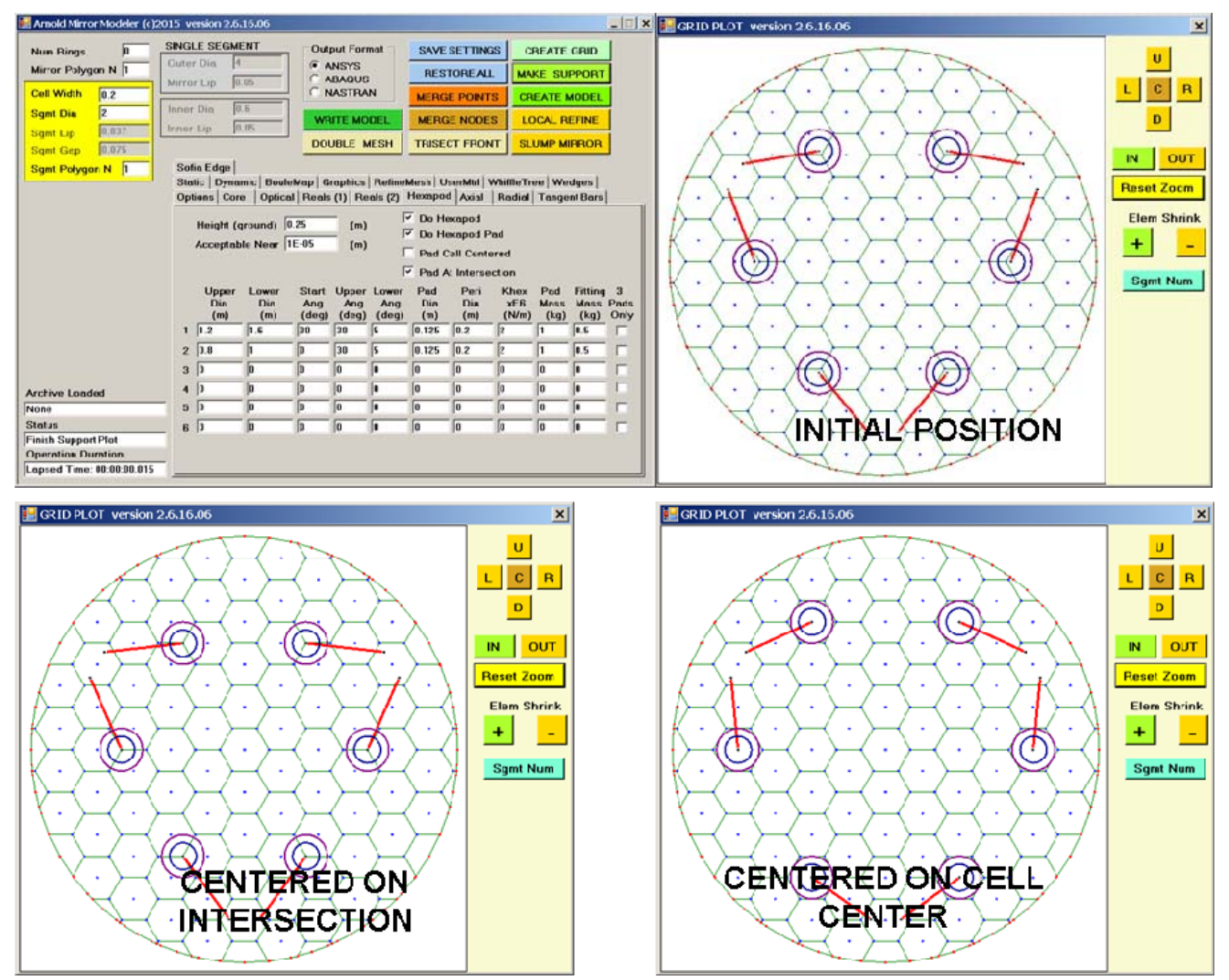

Figure 9. Automated pad relocation

\section{Off-axis optics}

With the improvement in diffraction performance with off-axis mirrors, a number of projects requested the ability to model large monolithic mirrors.
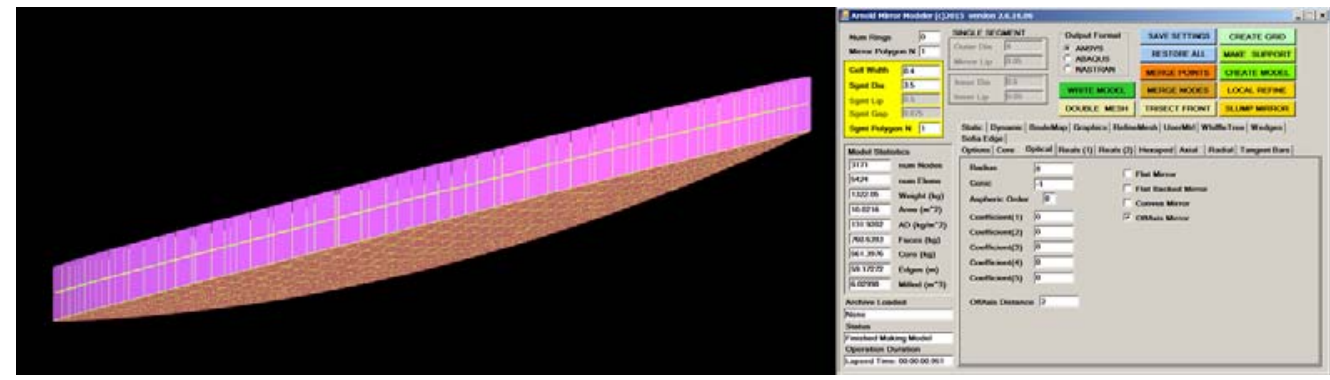

Figure 10 Off-axis optics 


\section{WHERE ARE WE GOING FROM HERE}

The program is continually under development, as new design ideas and materials or processes come to our attention, we endeavor to include the ability to model it. There is a number of planned or in-work improvements in the works.

Initial publication of the program is planned for the September 2015 time frame. Currently the distribution of the program is EXPORT ADMINISTRATION REGULATIONS restricted to US CITIZENS AND US ENTITIES.

Integration of the OUTPUT FILE formats with the OPTICAL PERFORMANCE ANALYSIS SYSTEMS under development for AMTD.

Improved BOND PAD modeling to include glue layer and actual pad geometry (a simplified mesh, suitable for preliminary design, not final).

\section{AKNOWLEGEMENTS}

This work has been supported by the AMTD project and Dr. H. Phil Stahl and the staff of ES31, ES22 and VP10 at Marshall Space Flight Center, Huntsville, Alabama.

A number of NASA interns have made major contributions to the development of the program, through their tireless efforts to break the code during testing and creative interruptions of the user's manual under development.

- Jacob Vehonsky

- $\quad$ Ryan M. Bevins

- Matthew Fitzgerald

- Rubin Jaca Rosa

- $\quad$ Erik Humfleet

\section{REFERENCES}

[1] Arnold, W. R., “AMTD DESIGN PROCESS”, NASA/SPIE Mirror Technology Days 2014, Albuquerque, NM (2014)

[2] Arnold, W. R., “Arnold Mirror Modeler Demonstration Video (30 min)”, NASA/SPIE Mirror Technology Days 2014, Albuquerque, NM (2014)

[3] Arnold, W. R., Etal., "Next-generation lightweight mirror modeling software”, SPIE Opto-mechanical Engineering 2013, San Diego, CA SPIE 8836-15 (2013)

[4] Arnold, W. R., Etal., "Integration of mirror design with suspension system using NASA's new mirror modeling software”, SPIE Opto-mechanical Engineering 2013, San Diego, CA SPIE 8836-17 (2013)

[5] Arnold, W. R., Etal. "Next-generation lightweight mirror modeling software”, NASA Mirror Tech Days 2013, Redondo Beach, CA (2013) 